PUPT-1932

ITEP-TH-23/00

NSF-ITP-00-43

hep-th/0005204

\title{
MONOPOLES AND STRINGS IN NONCOMMUTATIVE GAUGE THEORY
}

\author{
David J. Gross ${ }^{1}$, Nikita A. Nekrasov ${ }^{2}$ \\ ${ }^{1}$ Institute for Theoretical Physics, University of California Santa Barbara CA 93106 \\ ${ }^{2}$ Institute for Theoretical and Experimental Physics, 117259 Moscow, Russia \\ 2 Joseph Henry Laboratories, Princeton University, Princeton, New Jersey 08544 \\ e-mail: gross@itp.ucsb.edu, nikita@feynman.princeton.edu
}

\begin{abstract}
We study some non-perturbative aspects of noncommutative gauge theories. We find analytic solutions of the equations of motion, for noncommutative $\mathrm{U}(1)$ gauge theory, that describe magnetic monopoles with a finite tension string attached. These solutions are non-singular, finite and sourceless. We identify the string with the projection of a D-string ending on a D3-brane in the presence of a constant $B$-field.
\end{abstract}

$05 / 00$ 


\section{Introduction}

Recently there has been a revival of interest in field theories on noncommutative spaces [1] [2], especially those that emerge as various limits of $M$ theory compactifications [3]. The latest circumstances in which such theories were found involve D-branes in the presence of a background Neveu-Schwarz $B$-field [4] [ The interest in such theories is motivated by many analogies between noncommutative gauge theories and large $N$ ordinary non-abelian gauge theories [6] [7], and also by the many features that noncommutative field theories share with open string theory [8][7][9].

In this paper we study some non-perturbative dynamical objects in noncommutative gauge theory, specifically four dimensional gauge theory with an adjoint Higgs field. The theory depends on a dimensionfull parameter $\theta$ which enters the commutation relation between the coordinates of the space: $[x, x] \sim i \theta$. We treat only the bosonic fields, but these could be a part of a supersymmetric multiplet, with $\mathcal{N}=2$ supersymmetry or higher. Such field theories arise on the world volume of D3-branes in the presence of a background constant $B$-field along the D3-brane.

A D3-brane can be surrounded by other branes as well. For example, in the Euclidean setup, a D-instanton could approach the D3-brane. In fact, unless the D-instanton is dissolved inside the brane the combined system breaks supersymmetry [5]. The D3-D(-1) system can be rather simply described in terms of a noncommutative $U(1)$ gauge theory - the latter has instanton-like solutions [10]. However, it turns out that the "topology" of the combined system is non-trivial (despite the fact that the notion of a "point" on a noncommutative space makes very little sense, the non-triviality of topology can be detected), and it is this topology that supports the instantons [11].

Another, perhaps even more interesting situation, is that of a D-string that ends on a D3-brane. The endpoint of the D-string is a magnetic charge for the gauge field on the D3-brane. In the commutative case, in the absence of the $B$-field, the D-string is a straight line, orthogonal to the D3-brane. It projects onto the D3-brane in the form of a singular source, located at the point where the D-string touches the D3-brane. From the point of view of the D3-brane this is a Dirac monopole, with energy density that diverges at the origin.

The situation changes drastically when the $B$-field is turned on. One can trade a constant background $B$-field with spatial components for a constant background magnetic field. The latter pulls the magnetic monopoles with the constant force. As a consequence, 
the D-string bends [12], in order for its tension to compensate the magnetic force. It projects to the D3-brane as a half-line with finite tension. It is quite fascinating to see, as we shall explicitly verify, that the shadow of this string is seen by the noncommutative gauge theory. The $U(1)$ noncommutative gauge theory has a monopole solution, that is everywhere non-singular, and whose energy density localizes along a half-line, making up a semi-infinite string. We should stress that the non-singularity of the solution is the nonperturbative in $\theta$ property, it couldn't be seen by the expansion in $\theta$ around the Dirac monopole [13].

The fact that all the fields involved are non-singular, and that the solution is in fact a solution to the noncommutative version of the Bogomolny equations everywhere, makes us suspect that the string in the monopole solution is an intrinsic object of the gauge theory. As such, one could expect that the noncommutative gauge theory holographically describes strings as well. This statement is further supported by the fact that in the limit of very large $B$-field (the limit which must be well described by the non-commutative gauge theory [5]) the D-string almost lies on top of the D3-brane, practically dissolving in it.

Finally, by applying S-duality [14] one could map the solution we found into the solution describing the electric flux tube, represented by the fundamental string [15]. In this way one may hope to arrive at the description of the confining strings in the noncommutatve Yang-Mills theories. Notice however, that the S-duality maps the theory with the spatial noncommutativity to that of the temporal noncommutativity, with all its surprises [16][17], in addition to the strong coupling [14].

The outline of this paper is as follows. In Section 2 we consider some general features of noncommutative field theory, and discuss how it is convenient to work in the Fock space in which the coordinates are expresses as creation and annihilation operators. In Section 3 we construct the Green's function of the Laplace operator on noncommutative spaces, which illustrates the smearing of space induced by the noncommutativity of the coordinates. We also give a brief introduction to noncommuative gauge theories.

In Section 4 we set up the equations for BPS solutions of four dimension noncommutative gauge theories. We review Nahm's construction of commutative monopoles, exhibit the SU(2) monopole, as well as the Dirac monopole in this framework. Section 5 is devoted to the construction of the explicit solution of the BPS equations for the $U(1)$ noncommutative gauge theory coupled to a scalar field. The properties of the solution are analyzed in Section 6. We conclude, in Section 7, with a discussion of the implications of our analysis. 


\section{Acknowledgements.}

We would like to thank D. Bak, S. Cherkis, D. -E. Diaconescu, S. Giddings, A. Hashimoto, K. Hashimoto, N. Itzhaki, I. Klebanov, T. Piatina, A. Polyakov, A. Schwarz, S. Shatashvili, and K. Selivanov for discussions. Our research was partially supported by NSF under the grant PHY94-07194, in addition, research of NN was supported by Robert H. Dicke fellowship from Princeton University, partly by RFFI under grant 00-02-16530, partly by the grant 00-15-96557 for scientific schools. NN is grateful to ITP, UC Santa Barbara, CITUSC Center, and CGTP at Duke University for their hospitality during various stages of this work.

\section{Noncommutative Field Theory}

Consider space-time with coordinates $x^{i}, i=1, \ldots, d$ which obey the following commutation relations:

$$
\left[x^{i}, x^{j}\right]=i \theta^{i j}
$$

where $\theta^{i j}$ is a constant asymmetric matrix of rank $2 r \leq d$. By noncommutative space-time one means the algebra $\mathcal{A}_{\theta}$ generated by the $x^{i}$ satisfying (2.1), together with some extra conditions on the allowed expressions of the $x^{i}$. The elements of $\mathcal{A}_{\theta}$ can be identified with ordinary functions on $\mathbf{R}^{d}$, with the product of two functions $f$ and $g$ given by the Moyal formula (or star product):

$$
f \star g(x)=\left.\exp \left[\frac{i}{2} \theta^{i j} \frac{\partial}{\partial x_{1}^{i}} \frac{\partial}{\partial x_{2}^{j}}\right] f\left(x_{1}\right) g\left(x_{2}\right)\right|_{x_{1}=x_{2}=x}
$$

A field theory is defined as usual by constructing an action, say in the case of a scalar field theory,

$$
\mathcal{L}(\Phi)=\int d^{d} x\left[\partial_{i} \Phi \star \partial_{i} \Phi+V(\Phi)+\ldots\right]
$$

The symbol $\int d^{d} x$ is a notation for a trace, $\operatorname{Tr}$, on the algebra $\mathcal{A}_{\theta}$. When one works on compact noncommutative manifolds (compact manifolds, whose algebra of functions is deformed, e.g. by the techniques of [18]), for example the noncommutative torus, then the trace is the usual trace, i.e. the linear map $\mathcal{A}_{\theta} \rightarrow \mathbb{C}$, such that $\operatorname{Tr}[a, b]=0$. On $\mathbf{R}^{d}$ the notion of the trace is trickier, in particular the trace of the commutator may not vanish, just as the integral of a total derivative may not vanish. We shall encounter such effects 
in our discussion below, so instead of giving formal definitions at this point we shall treat explicit examples later.

The Lagrangian of a field theory involves derivatives. The derivative $\partial_{i}$ is the infinitesimal automorphism of the algebra (2.1):

$$
x^{i} \mapsto x^{i}+\varepsilon^{i},
$$

where $\varepsilon^{i}$ is a $c$-number. For the algebra (2.1) this automorphism is internal:

$$
\partial_{i} \Psi=i \theta_{i j}\left[\Psi, x^{j}\right]
$$

where $\theta_{i j}$ is the inverse of $\theta^{i j}$, namely $\theta_{i j} \theta^{j k}=\delta_{i}^{k}$. In contrast, on the torus generated by $U_{l}=\exp 2 \pi i x^{l}$, it is an outer automorphism. This difference is crucial in the analysis of noncommutative gauge theories.

By an orthogonal change of coordinates we can map the Poisson tensor $\theta_{i j}$ onto its canonical form:

$$
x^{i} \mapsto z_{a}, \bar{z}_{a}, \quad a=1, \ldots, r ; \quad y_{b}, \quad b=1, \ldots, d-2 r,
$$

so that:

$$
\begin{gathered}
{\left[y_{a}, y_{b}\right]=\left[y_{b}, z_{a}\right]=\left[y_{b}, \bar{z}_{a}\right]=0, \quad\left[z_{a}, \bar{z}_{b}\right]=2 \theta_{a} \delta_{a b}, \quad \theta_{a}>0} \\
d s^{2}=d x_{i}^{2}+d y_{b}^{2}=d z_{a} d \bar{z}_{a}+d y_{b}^{2} .
\end{gathered}
$$

Since $z(\bar{z})$ satisfy (up to a constant) the commutation relations of creation (annihilation) operators we can identify functions $f(x, y)$ with operator valued functions of the $y_{a}$ in the Fock space of the $r$ creation and annihilation operators (the operators in the Fock space are widely used in the studies of noncommutative theories and matrix models, for their applications to the latter see [19]):

$$
\alpha_{a}=z_{a} / \sqrt{2 \theta_{a}}, \quad \alpha_{a}^{\dagger}=\bar{z}_{a} / \sqrt{2 \theta_{a}}, \quad\left[\alpha_{a}, \alpha_{b}^{\dagger}\right]=\delta_{a b} .
$$

Since we shall be dealing with scale invariant theories in which the only scales will be the $\theta_{a}$ we shall set all $2 \theta_{a}=1$. When desired, the $\theta_{a}$ 's can be introduced by rescaling the coordinates, $z_{a} \rightarrow z_{a} / \sqrt{2 \theta_{a}}$. Let $\hat{n}_{a}=\alpha_{a}^{\dagger} \alpha_{a}$ be the $a$ 'th number operator.

The procedure that maps ordinary commutative functions onto operators in the Fock space acted on by $z_{a}, \bar{z}_{a}$ is called Weyl ordering and is defined by:

$$
f\left(x=\left(Z_{a}, \bar{Z}_{a}\right)\right) \mapsto \hat{f}\left(z_{a}, \bar{z}_{a}\right)=\int f(x) \frac{d^{2 r} x d^{2 r} p}{(2 \pi)^{2 r}} e^{i\left(\bar{p}_{a}\left(z_{a}-Z_{a}\right)+p_{a}\left(\bar{z}_{a}-\bar{Z}_{a}\right)\right)} .
$$


It is easy to see that

$$
\text { if } \quad f \mapsto \hat{f}, \quad g \mapsto \hat{g} \quad \text { then } \quad f \star g \mapsto \hat{f} \hat{g}
$$

A useful formula is for the matrix elements of $\hat{f}$ in the coherent state basis

$$
\langle\xi|\hat{f}| \eta\rangle=\int f(Z, \bar{Z}) \frac{d^{r} Z d^{r} \bar{Z}}{(2 \pi i)^{2 r}} e^{\xi \cdot \eta-2(\xi-\bar{Z}) \cdot(\eta-Z)}
$$

where $\langle\xi|$ and $|\eta\rangle$ are coherent states: $\left\langle\xi\left|=\left\langle\mathbf{0}\left|\exp \left(\xi_{a} z_{a}\right), \quad\right| \eta\right\rangle=\exp \left(\eta_{a} \bar{z}_{a}\right)\right| \mathbf{0}\right\rangle$. From (2.9) we can extract the matrix elements of $\hat{f}$ between the standard oscillator states by:

$$
\langle\mathbf{k}|\hat{f}| \mathbf{l}\rangle=\left.\frac{1}{\sqrt{\mathbf{k} ! \mathbf{l} !}} \partial_{\xi}^{\mathbf{k}} \partial_{\eta}^{\mathbf{l}}\right|_{\xi=\eta=0}\langle\xi|\hat{f}| \eta\rangle
$$

where $\mathbf{k}, \mathbf{l}$ are the vectors of the occupation numbers, e.g. $\mathbf{k}=\left(k_{1}, \ldots, k_{r}\right)$.

Given the operator $\hat{f}$, or its matrix elements, in the coherent state or occupation number basis, one can easily reconstruct the function $f(x)$ to which it corresponds. For example, consider the simplest case where $r=1, d=3$, that will be our interest below. Furthermore, consider functions that are axially symmetric. This means that $f(x)=$

$f\left(r, x_{3}\right)$, where $r=\sqrt{x_{1}^{2}+x_{2}^{2}}$; or equivalently that $\left\langle k\left|\hat{f}\left(x_{3}\right)\right| l\right\rangle=\delta_{k l} f_{l}\left(x_{3}\right)$. Then to reconstruct the function $f(x)$, from the matrix elements $f_{l}\left(x_{3}\right)$ one uses:

$$
f\left(r, x_{3}\right)=2 \sum_{l=0}^{\infty}(-1)^{l} f_{l}\left(x_{3}\right) L_{l}\left(4 r^{2}\right) e^{-2 r^{2}}
$$

where $L_{l}\left(4 r^{2}\right)$ are Laguerre polynomials.

\section{Scalar Field Green's functions}

An interesting property of noncommutative field theory is its similarity with lattice field theory, namely the noncommutativity of the coordinates introduces a smearing of space. We shall illustrate this similarity by examining the Green's functions of the Laplace operator on noncommutative space-time. 


\subsection{Sources}

Consider the noncommutative version of the equation for the Green's function $\Delta_{x} G\left(x, x^{\prime}\right)=\delta\left(x-x^{\prime}\right)$. Recalling (2.4), the Laplace operator can be rewritten as follows:

$$
\hat{\Delta}=\frac{\partial^{2}}{\partial y_{b}^{2}}-4 \theta_{a}^{-2}\left[\left[\cdot, z_{a}\right], \bar{z}_{a}\right]
$$

Thus the noncommutative equation for the Green's function is

$$
\frac{\partial^{2}}{\partial y_{b}^{2}} \hat{G}\left(z, \bar{z}, y ; z^{\prime}, \bar{z}^{\prime}, y^{\prime}\right)-4\left[\left[\hat{G}\left(z, \bar{z}, y ; z^{\prime}, \bar{z}^{\prime}, y^{\prime}\right), z_{a}\right], \bar{z}_{a}\right]=\hat{\delta}\left(z, \bar{z} ; z^{\prime}, \bar{z}^{\prime}\right) \delta\left(y-y^{\prime}\right)
$$

where we have introduced two copies of the algebra $\mathcal{A}_{\theta}$, generated by $z_{a}, \bar{z}_{a}, z_{a}^{\prime}, \bar{z}_{a}^{\prime}$ and $\hat{\delta}, \hat{G}$ are operators in the tensor product of two Fock spaces

$$
\mathcal{H}_{1,2}=\mathcal{H}_{1} \otimes \mathcal{H}_{2}
$$

spanned by $\left|\mathbf{l}_{1}, \mathbf{l}_{2}\right\rangle=\left|\mathbf{l}_{1}\right\rangle \otimes\left|\mathbf{l}_{2}\right\rangle$.

The expression for the delta function, $\hat{\delta}\left(z, \bar{z} ; z^{\prime}, \bar{z}^{\prime}\right)$, is now easy to obtain directly in the coherent state basis, using the (tensor product form of) (2.9). In terms of $\left|\eta_{1}, \eta_{2}\right\rangle=$ $\left|\eta_{1}\right\rangle \otimes\left|\eta_{2}\right\rangle$ we have

$$
\begin{aligned}
\left\langle\xi_{1}, \xi_{2}|\hat{\delta}| \eta_{1}, \eta_{2}\right\rangle= & \int e^{\xi_{1} \cdot \eta_{1}+\xi_{2} \cdot \eta_{2}-2\left(\xi_{1}-\bar{Z}\right) \cdot\left(\eta_{1}-Z\right)-2\left(\xi_{2}-\bar{Z}\right) \cdot\left(\eta_{2}-Z\right)} d^{r} Z d^{r} \bar{Z} \\
& =e^{\xi_{1} \cdot \eta_{2}+\xi_{2} \cdot \eta_{1}}
\end{aligned}
$$

The matrix elements of $\delta$ in the occupation number basis are:

$$
\left\langle\mathbf{k}_{1,2}|\hat{\delta}| \mathbf{l}_{1,2}\right\rangle=\delta_{\mathbf{k}_{1}, \mathbf{l}_{2}} \delta_{\mathbf{k}_{2}, \mathbf{l}_{1}}
$$

Thus $\hat{\delta}$ is a permutation operator $P: \mathcal{H} \otimes \mathcal{H} \rightarrow \mathcal{H} \otimes \mathcal{H}, P\left(e_{1} \otimes e_{2}\right)=e_{2} \otimes e_{1}$, and squares to the identity operator $P^{2}=I d$. It is easy to verify that $\hat{\delta}$ satisfies the defining property of the delta-function, namely

$$
\operatorname{Tr}_{x}^{\prime}\left[\hat{\delta}\left(x, x^{\prime}\right) \hat{f}\left(x^{\prime}\right)\right]=\hat{f}(x)
$$

What is the noncommutative version of a source localized at the origin, namely $\delta^{2 r}(x)$ ? Using (2.9) we see that

$$
\langle\xi|\hat{\delta}| \eta\rangle=\int \delta^{2}(Z) \frac{d^{r} Z d^{r} \bar{Z}}{(2 \pi i)^{2 r}} e^{\xi \cdot \eta-2(\xi-\bar{Z}) \cdot(\eta-Z)}=e^{-\xi \cdot \eta}
$$


or in the occupation number basis:

$$
\langle\mathbf{k}|\hat{\delta}| \mathbf{l}\rangle=\delta_{\mathbf{k}, \mathbf{l}}(-1)^{|\mathbf{k}|}, \quad \hat{\delta}=(-1)^{\hat{\mathbf{n}}}
$$

with $|\mathbf{k}|=\sum_{a} k_{a}, \hat{\mathbf{n}}=\sum_{a} \hat{n}_{a}$. In this way the delta function becomes an operator in the Fock space with the spectrum of the form of the diffraction rings. Note that $(\hat{\delta}(x))^{2}=I d$, which is the transform of the constant function.

Alternatively we can relate (3.4) to (3.7). by passing to the center-of-mass frame:

$$
z^{c}=\frac{1}{\sqrt{2}}\left(z+z^{\prime}\right), z^{r}=\frac{1}{\sqrt{2}}\left(z-z^{\prime}\right)
$$

and similarly for $\bar{z}, \bar{z}^{\prime}$. The expression (3.7) is written in the number basis for the operators $z^{r}, \bar{z}^{r}$. The transformation (3.8) is a unitary one:

$$
\begin{aligned}
& S z^{r} S^{\dagger}=z, \quad S z^{c} S^{\dagger}=z^{\prime}, \\
& S z S^{\dagger}=z^{c}, \quad S z^{\prime} S^{\dagger}=-z^{r} \\
& S=\exp \frac{\pi}{4}\left(\bar{z}^{\prime} \cdot z-\bar{z} \cdot z^{\prime}\right)
\end{aligned}
$$

It is easy to check that $S P=P S^{\dagger}$. Therefore $S P S^{\dagger}=S^{2} P$. Now, consider $U=S^{2}$. It acts as follows:

$$
U z U^{\dagger}=z^{\prime}, \quad U z^{\prime} U^{\dagger}=-z
$$

Let us now apply the $S$ transformation to the delta function:

$$
S P S^{\dagger}\left|\mathbf{l}_{1}\right\rangle \otimes\left|\mathbf{l}_{2}\right\rangle=U\left|\mathbf{l}_{2}\right\rangle \otimes\left|\mathbf{l}_{1}\right\rangle=(-1)^{\left|\mathbf{l}_{1}\right|}\left|\mathbf{l}_{1}\right\rangle \otimes\left|\mathbf{l}_{2}\right\rangle
$$

i.e. we get complete agreement with (3.7).

Thus in the noncommutative case we cannot construct a truly localized source. The transform of $\delta^{2 r}(x)$, in which the noncommuting coordinates are all localized at the origin, is spread out over all of space. The most localized source we can construct in the noncommutative case is a Gaussian wave packet $D(x)=\exp (-2 Z \bar{Z})$, whose transform is

$$
\hat{D}=|\mathbf{0}\rangle\langle\mathbf{0}|, \quad\langle\xi|\hat{D}| \eta\rangle=1, \quad\langle\mathbf{k}|\hat{D}| \mathbf{l}\rangle=\delta_{\mathbf{k}, \mathbf{l}} \delta_{\mathbf{l}, \mathbf{0}}
$$

One can also develop the similar analysis for finite lattices, in which case one gets the finite matrix versions of the operators (3.7) (3.4) (see [20]). 


\subsection{Green's functions}

We now consider the Laplace equation for the Green's function, $\hat{G}$. Consider a function (an element of $\mathcal{A}_{\theta}$ ) that commutes with all $N_{a}$ 's. In the commutative language this means that the functions we wish to look at are invariant under rotations of the $z_{a}, \bar{z}_{a}$ two-planes. We take $\hat{G}$ to be such a function. On such functions the Laplacian acts as follows:

$$
\Delta G_{\mathbf{n}}=\frac{\partial^{2}}{\partial y_{b}^{2}} G_{\mathbf{n}}+4 \sum_{a}\left(-\left(2 n_{a}+1\right) G_{\mathbf{n}}+\left(n_{a}+1\right) G_{\mathbf{n}+\mathbf{e}_{a}}+n_{a} G_{\mathbf{n}-\mathbf{e}_{a}}\right)
$$

where

$$
\mathbf{n}=\left(n_{1}, \ldots, n_{r}\right), \quad \mathbf{e}_{a}=(0,0, \ldots, \underset{\widehat{a}}{1}, \ldots, 0), \quad\left\langle\mathbf{n}|\hat{G}| \mathbf{n}^{\prime}\right\rangle=\delta_{\mathbf{n}^{\prime}, \mathbf{n}} G_{\mathbf{n}}
$$

The formula (3.12) requires the following comment: when evaluating the right hand side of (3.12) the number operators $n_{a}$ must be evaluated first, so that if some of the $n_{a}$ 's vanish the whole expression $n_{a} G_{\mathbf{n}-\mathbf{e}_{a}}$ must be set to zero, no matter how singular the analytic expression for $G_{\mathbf{n}-\mathbf{e}_{a}}$ may look.

One can also rewrite the Laplacian (3.12) using the finite difference operators: $\mathcal{D}_{a}, \mathcal{N}_{a}$ :

$$
\begin{gathered}
\mathcal{D}_{a} G_{\mathbf{n}}=G_{\mathbf{n}}-G_{\mathbf{n}-\mathbf{e}_{a}}, \quad \mathcal{N}_{a} G_{\mathbf{n}}=\left(n_{a}+1\right) G_{\mathbf{n}+\mathbf{e}_{a}} \\
\Delta=\frac{\partial^{2}}{\partial y_{b}^{2}}+4 \mathcal{D}_{a} \mathcal{N}_{a} \mathcal{D}_{a}
\end{gathered}
$$

The operators $\mathcal{N}, \mathcal{D}$ form a Heisenberg algebra:

$$
\left[\mathcal{D}_{a}, \mathcal{N}_{b}\right]=\delta_{a b}
$$

Let us compare the expressions $(3.12),(3.15)$ to their commutative analogues. Let $y_{b}, Z_{a}, \bar{Z}_{a}$ denote the coordinates on the commutative space-time with the metric

$$
d s^{2}=d y_{b}^{2}+\frac{1}{2} d Z_{a} d \bar{Z}_{a}
$$

and consider functions that depend only on $y_{b}$ and $R_{a}=\left|Z_{a}\right|^{2}$. On such a function, say $\mathcal{G}$, the Laplacian acts as follows:

$$
\Delta \mathcal{G}=\frac{\partial^{2}}{\partial y_{b}^{2}} \mathcal{G}+4 \sum_{a} \frac{\partial}{\partial R_{a}} R_{a} \frac{\partial}{\partial R_{a}} \mathcal{G}
$$


The operators $R_{a}$ and $\partial_{R_{b}}$ form the same Heisenberg algebra (3.16)as $\mathcal{N}, \mathcal{D}$. Consequently, by mapping the representation (3.14) to the standard representation of the Heisenberg algebra acting on functions of $R_{a}$, we can map the Green's function of the noncommutative Laplacian to that of the commutative one.

Let us note, however, that the algebra (3.16) is represented by functions on the whole space $\mathbf{R}^{r}$, whereas the variables $R_{a}$, by definition, must be positive. The same comment applies to the variables $n_{a} \geq 0$. This boundnessness of the domain in the definition of these functions produces the source terms in the Laplace (or other) equations that they obey.

Now construct the Laplace transform of the function $\mathcal{G}$ :

$$
\tilde{\mathcal{G}}(t)=\int_{0}^{\infty} \prod_{a} d R_{a} e^{-t_{a} R_{a}} \mathcal{G}\left(R_{a}\right) .
$$

At the same time we construct the generating function associated with $G_{\mathbf{n}}$ :

$$
\hat{G}(t)=\sum_{\mathbf{n}} \prod_{a}\left(1-t_{a}\right)^{n_{a}} G_{\mathbf{n}}
$$

It is easy to see that the Laplacian operators acting on both $\tilde{\mathcal{G}}(t)$ and on $\hat{G}(t)$, are mapped to the same operator:

$$
\hat{\Delta}=\frac{\partial}{\partial y_{b}^{2}}-4 t_{a} \frac{\partial}{\partial t_{a}} t_{a},
$$

(as long as we assume that the functions don't grow too fast at infinity or at zero) In this way we define a map from functions of continuous $R$ coordinates to functions of discrete n:

$$
G_{\mathbf{n}}=\int_{0}^{\infty} \mathcal{G}_{R} \prod_{a} \frac{R_{a}^{n_{a}}}{n_{a} !} d R_{a} e^{-R_{a}} .
$$

Note that as $\mathbf{n} \rightarrow \infty$ the saddle point approximation gives

$$
G_{\mathbf{n}} \sim \mathcal{G}_{R_{a}=n_{a}}
$$

We can use this to construct the noncommutative version of the Green's function. For example, take $d=2 r+1$, then the $2 r+1$ - dimensional Green's function

$$
\mathcal{G}(y, R)=\frac{1}{\left(y^{2}+\sum_{a} R_{a}\right)^{r+\frac{1}{2}}},
$$


transforms into:

$$
G_{\mathbf{n}}=\int_{0}^{\infty} \mathrm{d} s \frac{s^{r-\frac{3}{2}}}{(1+s)^{|\mathbf{n}|+\mathbf{r}}} e^{-s y^{2}}, \quad|\mathbf{n}|=\sum_{\mathbf{a}} \mathbf{n}_{\mathbf{a}} .
$$

The noncommutative function is everywhere non-singular:

$$
G_{0}(y) \sim-\sqrt{\pi}\left(2 \frac{\left(r-\frac{3}{2}\right) !}{(r-2) !}+|y|+\ldots\right), \quad y \rightarrow .0
$$

Thus the map renders functions smoother at the origin.

Another example is when $d=2 r$. Then we have:

$$
\begin{gathered}
\mathcal{G}(R)=\frac{1}{\left(\sum_{a} R_{a}\right)^{r-1}} \\
G_{\mathbf{n}}=\int_{0}^{1} \mathrm{~d} \lambda \lambda^{r-2}(1-\lambda)^{|\mathbf{n}|}=\frac{(r-2) !|\mathbf{n}| !}{(|\mathbf{n}|+r-1) !} .
\end{gathered}
$$

which is also non-singular everywhere (for $r>1$ ). In two dimensions $(r=1)$ we get:

$$
\mathcal{G}(R)=\log (\mu R) \Rightarrow G_{n}=\log \mu+\psi(n+1)=\log \mu-C+\sum_{k=1}^{N} \frac{1}{k}
$$

The formula (3.24) is not applicable here since it gives a logarithmically divergent integral, of purely infrared origin. However, the divergence is $n$ independent, so that it affects $G_{n}$ by an additive constant, i.e. by a zero mode of the Laplacian. The presence of the divergence is reflected in the fact that the cutoff, $\log \mu$, in the commutative Green's function appears in the noncommutative formula (3.25).

The commutative Green's function $\mathcal{G}(R)$ solved Laplace's equation with a delta function source:

$$
\Delta \mathcal{G}(R)=\delta^{d}(x)
$$

Let us see what the source is equal to now. For simplicity let us work in even number of dimensions, $G_{\mathbf{n}}=G(|\mathbf{n}|)$ :

$$
\frac{1}{4} \Delta G(n)=(n+r) G(n+1)+n G(n-1)-(2 n+r) G(n)=-\int_{0}^{1} \mathrm{~d}\left(\lambda^{r}(1-\lambda)^{n}\right)=-\delta_{n, 0} .
$$

So the source got smoothed out:

$$
\delta^{d}(x)=\prod \delta\left(R_{a}\right) \prod \delta\left(y_{b}\right) \mapsto \prod \delta_{N_{a}, 0} \prod \delta\left(y_{b}\right)
$$


In this way the noncommutativity of the space-time looks similar to the lattice regularization (although in the spherical rather then Cartesian way). However, by the above analysis, the formula (3.26) means that we have ended up with the Gaussian source $D$ as in (3.11).

On the other hand, the solution to the equation

$$
\Delta \hat{G}=\hat{\delta}
$$

with the localized delta function source is also easy to produce: one simply applies the map (2.7) to the ordinary Green's function $\mathcal{G}_{R}$. It is amusing that the result is close to the formula (3.24), namely the Green's function is again diagonal in the eigenbasis of the occupation number operators $n_{a}$ and depends only on $n=|\mathbf{n}|$ :

$$
\hat{G}_{\mathbf{n}}=\hat{G}(n)=\int_{0}^{2} \mathrm{~d} \lambda \lambda^{r-2}(1-\lambda)^{n} .
$$

However, (3.28) and (3.24) differ considerably. In four dimensions, $r=2$, the difference is striking:

$$
G(n)=\frac{1}{n+1}, \quad \hat{G}(n)=\frac{1+(-1)^{n}}{n+1} .
$$

What is also striking is the failure of the classical limit for $r>2$ : one might expect that, for large $n$, the Green's function $\hat{G}(n)$ would go over to its classical counterpart $\mathcal{G}_{R} \sim \frac{1}{n^{r-1}}$. The integral (3.24) indeed has this property - the integrand is peaked at $\lambda=0$ and the saddle point gives precisely the expected asymptotics. But the integral (3.28) has another saddle point at $\lambda=2$ which yields the leading asymptotics for $r>2$

$$
\hat{G}(n) \sim \frac{(-1)^{n} 2^{r-2}}{n+1}, \quad n \rightarrow \infty .
$$

The lesson to be drawn from here is that highly localized distributions (the delta function is such a distribution) become the operators spread out over all the Fock space that they act in, while the operators whose range is comparatively small (such as the Gaussian) correspond in fact to the distributions with finite support of order $\sim \sqrt{\theta}$.

Finally, to construct the Green's function $\hat{G}\left(x-x^{\prime}\right)$ we have to use the formula:

$$
\hat{G}\left(x-x^{\prime}\right)=S^{\dagger}\left(\hat{G}(x) \otimes \operatorname{Id}_{x^{\prime}}\right) S,
$$

which gives (for $d=2 r$ ):

$$
G\left(x-x^{\prime}\right)=\int_{0}^{2} \mathrm{~d} \lambda \lambda^{r-2}(1-\lambda)^{\frac{1}{2}\left(z-z^{\prime}\right) \cdot\left(\bar{z}-\bar{z}^{\prime}\right)} .
$$




\subsection{Gauge theory on noncommutative space}

In an ordinary gauge theory with gauge group $G$ the gauge fields are connections in some principal $G$-bundle. The matter fields are the sections of the vector bundles with the structure group $G$. Noncommutative vector bundles are defined as projective modules over the algebra $\mathcal{A}_{\theta}$. This definition captures the following two properties of ordinary vector bundles: i) the sections of the bundle can be multiplied by functions on the base manifold - in this way the space of sections is acted on (linearly) by the space of functions - the definition of a module; ii) every vector bundle can be made trivial by the appropriate addition of another vector bundle - this is the definition of the projective module - it becomes free (equals to a direct sum of several copies of the algebra $\mathcal{A}_{\theta}$ ) when we add another module.

Now suppose we are given a module $M$ over the algebra $\mathcal{A}_{\theta}$. In the noncommutative case there are two types of modules, left and right. The elements $m_{1}$ of the left module are multiplied by the elements $a$ of the algebra from the left, while the elements of the right module are multiplied from the right:

$$
a: m_{\mathbf{l}} \mapsto a m_{\mathbf{l}}, \quad m_{\mathbf{r}} \mapsto m_{\mathbf{r}} a
$$

The left module over an algebra $\mathcal{A}$ is a right module over the algebra $\mathcal{A}^{\prime}$ which is obtained from $\mathcal{A}$ by reversing the order of multiplication:

$$
a \star^{\prime} b=b \star a .
$$

The notion of the left/right modules is analogous to the notion of chiral matter fields.

The connection $\nabla$ is the operator

$$
\nabla: \mathbf{R}^{d} \times M \rightarrow M, \quad \nabla_{\varepsilon}(m) \in M, \quad \varepsilon \in \mathbf{R}^{d}, m \in M
$$

where $\mathbf{R}^{d}$ denotes the commutative vector space, the Lie algebra of the automorphism group generated by (2.3). The connection is required to obey the Leibnitz rule:

$$
\begin{aligned}
& \nabla_{\varepsilon}\left(a m_{\mathbf{l}}\right)=\varepsilon^{i}\left(\partial_{i} a\right) m_{\mathbf{l}}+a \nabla_{\varepsilon} m_{\mathbf{l}} \\
& \nabla_{\varepsilon}\left(m_{\mathbf{r}} a\right)=m_{\mathbf{r}} \varepsilon^{i}\left(\partial_{i} a\right)+\left(\nabla_{\varepsilon} m_{\mathbf{r}}\right) a
\end{aligned}
$$

As usual, one defines the curvature $F_{i j}=\left[\nabla_{i}, \nabla_{j}\right]$ - the operator $\Lambda^{2} \mathbf{R}^{d} \times M \rightarrow M$ which commutes with the multiplication by $a \in \mathcal{A}_{\theta}$. In other words, $F_{i j} \in \operatorname{End}_{\mathcal{A}}(M)$. If 
the right (left) module $M$ is free, i.e. it is a sum of several copies of the algebra $\mathcal{A}$ itself, then the connection $\nabla_{i}$ can be written as

$$
\nabla_{i}=\partial_{i}+A_{i}
$$

where $A_{i}$ is the operator of the left (right) multiplication by the matrix with $\mathcal{A}$-valued entries. In the same operator sense the curvature obeys the standard identity:

$$
F_{i j}=\partial_{i} A_{j}-\partial_{j} A_{i}+A_{i} A_{j}-A_{j} A_{i}
$$

Given the module $M$ one can multiply it by a free module $\mathcal{A}^{\oplus N}$ to make it a module over an algebra $\operatorname{Mat}_{N \times N}(\mathcal{A})$ of matrices with elements from $\mathcal{A}$. In the non-abelian gauge theory over $\mathcal{A}_{\theta}$ we are interested in projective modules over $\operatorname{Mat}_{N \times N}\left(\mathcal{A}_{\theta}\right)$. If the algebra $\mathcal{A}_{\theta}$ (or perhaps its subalgebra) has a trace, $\operatorname{Tr}$, then the algebra $\operatorname{Mat}_{N \times N}\left(\mathcal{A}_{\theta}\right)$ has a trace given by the composition of a usual matrix trace and Tr.

It is a peculiar property of the noncommutative algebras that the algebras $\mathcal{A}$ and $\operatorname{Mat}_{N \times N}(\mathcal{A})$ have much in common. These algebras are called Morita equivalent and under some additional conditions the gauge theories over $\mathcal{A}$ and over $\operatorname{Mat}_{N}(\mathcal{A})$ are also equivalent. This phenomenon is responsible for the similarity between the "abelian noncommutative" and "non-abelian commutative" theories.

\section{Monopoles and Instantons}

\subsection{Lagrangian and couplings}

After the preparations of the previous section the Lagrangian for the gauge theory is given by:

$$
\mathcal{L}(A)=-\frac{1}{4 g_{\mathrm{YM}}^{2}} \sum_{i, j} \operatorname{Tr}\left[\nabla_{i}, \nabla_{j}\right]^{2} .
$$

If additional charged matter fields are present (elements $\Phi$ of a module $M$ ) then the Lagrangian becomes:

$$
\mathcal{L}(A, \Phi)=\mathcal{L}(A)+\sum_{i} \operatorname{Tr} \nabla_{i} \Phi \nabla_{i} \Phi+\ldots
$$

The equations of motion following from the Lagrangian (4.1) are:

$$
\sum_{i}\left[\nabla_{i}, F_{i j}\right]=0
$$


In four dimensions, $d=4$, the Euclidean version of (4.3) can be solved by solving the first order instanton equations:

$$
F_{i j}= \pm \frac{1}{2} \epsilon_{i j k l} F_{k l}
$$

as follows from the Bianci identity, which holds irrespectively of the commutativity:

$$
\left[\nabla_{i}, F_{k l}\right]+\left[\nabla_{k}, F_{l i}\right]+\left[\nabla_{l}, F_{i k}\right]=0
$$

Introduce the complex coordinates: $z_{1}=x_{1}+i x_{2}=x_{+}, z_{2}=x_{3}+i x_{4}$. The instanton equations read:

$$
\begin{aligned}
F_{z_{1} z_{2}} & =0 \\
F_{z_{1} \bar{z}_{1}}+F_{z_{2} \bar{z}_{2}} & =0 .
\end{aligned}
$$

The first equation in (4.6) can be solved (locally) as follows:

$$
A_{\bar{z}_{a}}=\xi^{-1} \bar{\partial}_{\bar{z}_{a}} \xi, \quad A_{z_{a}}=-\partial_{z_{a}} \xi \xi^{-1}
$$

with $\xi$ a Hermitian matrix. Then the second equation in (4.6) becomes Yang's equation:

$$
\sum_{a=1}^{2} \bar{\partial}_{z_{a}}\left(\partial_{z_{a}} \xi^{2} \xi^{-2}\right)=0
$$

This ansatz works in the noncommutative setup as well [10].

If we look for the solutions to (4.6), that are invariant under translations in the 4'th direction then we will find the monopoles of the gauge theory with an adjoint scalar Higgs field, where the role of the Higgs field is played by the component $A_{4}$ of the gauge field. The equations (4.6) in this case are called the Bogomolny equations, and they can be analyzed in the commutative case via Nahm's ansatz [21].

The action (4.1) becomes the energy functional for the coupled gauge-adjoint Higgs system:

$$
\mathcal{E}=\frac{1}{4 g_{\mathrm{YM}}^{2}} \int d^{3} x \sqrt{\operatorname{det} G} \operatorname{Tr}\left(G^{i i^{\prime}} G^{j j^{\prime}} F_{i j} \star F_{i^{\prime} j^{\prime}}+G^{i j} \nabla_{i} \Phi \star \nabla_{j} \Phi\right)
$$

where for the sake of generality we have introduced a constant metric $G_{i j}$. Open and closed string moduli.

(4.9) emerges in the decoupling limit of a D3-brane in the Type IIB string theory in a background with a constant Neveu-Schwarz B-field. Let us recall the relation of the parameters of the actions (4.1), (4.9) and the string theory parameters, before taking the Seiberg-Witten limit [5]. 
We start with the D3-brane whose worldvolume is occupying the 0123 directions, and turn on a $B$-field:

$$
\frac{1}{2} B d x^{1} \wedge d x^{2}
$$

The indices $i, j$ below will run from 1 to 3 . We assume that the closed string metric $g_{i j}$ is flat, and the closed string coupling $g_{s}$ is small. According to [5] the gauge theory on the D3-brane is described by a Lagrangian, which, when restricted to time-independent fields, coincides with (4.9), whose parameters

$$
G_{i j}, \theta^{i j}, g_{\mathrm{YM}}^{2}
$$

are related to

$$
g_{i j}, B_{i j}, g_{s}
$$

as follows:

$$
\begin{aligned}
& G_{i j}=g_{i j}-\left(2 \pi \alpha^{\prime}\right)^{2}\left(B g^{-1} B\right)_{i j} \\
& \theta^{i j}=-\left(2 \pi \alpha^{\prime}\right)^{2}\left(\frac{1}{g+2 \pi \alpha^{\prime} B} B \frac{1}{g-2 \pi \alpha^{\prime} B}\right)^{i j} \\
& g_{\mathrm{YM}}^{2}=2 \pi g_{s}\left(\operatorname{det}\left(1+2 \pi \alpha^{\prime} g^{-1} B\right)\right)^{\frac{1}{2}} .
\end{aligned}
$$

Suppose the open string metric is Euclidean: $G_{i j}=\delta_{i j}$, then (4.10), (4.11) imply:

$$
g=d x_{3}^{2}+\frac{\left(2 \pi \alpha^{\prime}\right)^{2}}{\left(2 \pi \alpha^{\prime}\right)^{2}+\theta^{2}}\left(d x_{1}^{2}+d x_{2}^{2}\right), \quad B=\frac{\theta}{\left(2 \pi \alpha^{\prime}\right)^{2}+\theta^{2}}
$$

and

$$
g_{s}=g_{\mathrm{YM}}^{2} \frac{\alpha^{\prime}}{\sqrt{\left(2 \pi \alpha^{\prime}\right)^{2}+\theta^{2}}} .
$$

The Seiberg-Witten limit is achieved by taking $\alpha^{\prime} \rightarrow 0$ with $G, \theta, g_{\mathrm{YM}}^{2}$ kept fixed.

In this limit the effective action of the D3-brane theory will become that of the (super)Yang-Mills theory on a noncommutative space $\mathcal{A}_{\theta}$. The relevant part of the energy functional is:

$$
\mathcal{E}=\frac{1}{2 g_{\mathrm{YM}}^{2}} \int d^{3} x \operatorname{Tr}\left(B_{i} \star B_{i}+\nabla_{i} \Phi \star \nabla_{i} \Phi\right)
$$

where

$$
B_{i}=\frac{i}{2} \varepsilon_{i j k} F_{j k}
$$

The fluctuations of the D3-brane in some distinguished transverse direction (which we called $\Phi)$ are described by the dynamics of the Higgs field. 
As in the ordinary, commutative case, one can rewrite 4.14) as a sum of a total square and a total derivative:

$$
\mathcal{E}=\frac{1}{2 g_{\mathrm{YM}}^{2}} \int d^{3} x \operatorname{Tr}\left(\nabla_{i} \Phi \pm B_{i}\right)^{2} \mp \partial_{i} \operatorname{Tr}\left(B_{i} \star \Phi+\Phi \star B_{i}\right)
$$

The total derivative term depends only on the boundary conditions. So, to minimize the energy given boundary conditions we should solve the Bogomolny equations:

$$
\nabla_{i} \Phi= \pm B_{i}, \quad i=1,2,3
$$

\subsection{Nahm's construction for commutative monopoles}

To begin with, we review the techniques which have been used in the commutative case. Specifically, Bogomolny equations take the form:

$$
\nabla_{i} \Phi+B_{i}=0, \quad i=1,2,3
$$

The boundary condition is that at the spatial infinity the Higgs field approaches a constant, corresponding to the Higgs vacuum. In the case of $S U(2)$ this means that locally on the two-sphere at infinity:

$$
\phi(x) \sim \operatorname{diag}\left(\frac{a}{2},-\frac{a}{2}\right) .
$$

The solutions are classified by the magnetic charge $k$. By virtue of the equation (4.18) the monopole charge can be expressed as the winding number which counts how many times the two-sphere $\mathbf{S}_{\infty}^{2}$ at infinity is mapped to the coset space $S U(2) / U(1) \approx \mathbf{S}^{2}$ of the abelian subgroups of the gauge group.

Nahm [21] constructs solutions to the monopole equations as follows. Consider the matrix differential operator on the interval $I$ with the coordinate $z$ :

$$
-i \Delta=\partial_{z}+\mathcal{T}_{i} \sigma_{i}
$$

where

$$
\mathcal{T}_{i}=T_{i}(z)+x_{i}
$$

$x_{i}$ are the coordinates in the physical space $\mathbf{R}^{3}$, and the $k \times k$ matrices $T_{i}(z)=T_{i}^{\dagger}(z)$ obey Nahm's equations:

$$
\partial_{z} T_{i}=i \varepsilon_{i j k} T_{j} T_{k}
$$


with certain boundary conditions. We take $I=(-a / 2, a / 2)$ where $a$ is given in (4.19). At $z \rightarrow z_{0}, z_{0}= \pm a / 2$ we require that :

$$
T_{i} \sim \frac{t_{i}}{z-z_{0}}+\text { reg., } \quad\left[t_{i}, t_{j}\right]=i \varepsilon_{i j k} t_{k}
$$

i.e. the residues $t_{i}$ must form a $k$-dimensional representation of $S U(2)$ (irreducible if the solution is to be non-singular).

Then one looks for the fundamental solution to the equation:

$$
-i \Delta^{\dagger} \Psi(z)=\partial_{z} \Psi-\mathcal{T}_{i} \sigma_{i} \Psi=0
$$

where

$$
\Psi=\left(\begin{array}{c}
\Psi_{+} \\
\Psi_{-}
\end{array}\right),
$$

and $\Psi_{ \pm}$are $k \times 2$ matrices ( $k$ is the monopole charge, and 2 is for $S U(2)$ ), which must be finite at $z= \pm a / 2$ and normalized so that:

$$
\int d z \Psi^{\dagger} \Psi=\mathbf{1}_{2 \times 2} .
$$

(this $2 \times 2$ is again for $S U(2)$.)

Then:

$$
\begin{aligned}
A_{i} & =\int d z \Psi^{\dagger} \partial_{i} \Psi \\
\Phi & =\int d z z \Psi^{\dagger} \Psi .
\end{aligned}
$$

Notice that the interval $I$ could be $\left(a_{1}, a_{2}\right)$ instead of $(-a / 2,+a / 2)$. The only formula that is not invariant under shifts of $z$ is the expression (4.26) for $\phi$. By shifting $\phi$ by a scalar $\left(a_{1}+a_{2}\right) / 2$ we can make it traceless and map $I$ back to the form we used above.

\subsection{Nahm's equations from the D-string point of view}

The meaning of the Nahm's equations becomes clearer in the D-brane realization of gauge theory and the D-string construction of monopoles. The endpoint of a fundamental string touching a D3-brane looks like an electric charge for the $U(1)$ gauge field on the brane. By S-duality, a D-string touching a D3-brane creates a magnetic monopole. If one starts with two parallel D3-branes, seperated by distance $a$ between them, one is studying the $U(2)$ gauge theory, Higgsed down to $U(1) \times U(1)$, where the vev of the Higgs field is

$$
\Phi=\left(\begin{array}{cc}
a_{1} & 0 \\
0 & a_{2}
\end{array}\right)
$$


One can suspend a D-string between these two D3-branes, or a collection of $k$ parallel D-strings. These would correspond to a charge $k$ magnetic monopole in the Higgsed $U(2)$ theory. The BPS configurations of these D-strings are described the corresponding selfduality equations in the $1+1$ dimensional $U(k)$ gauge theory on the worldsheet of these strings [22]. The equations (4.22) are exactly these BPS equations. The presence of the D3-branes is reflected in the boundary conditions (4.23). The matrices $T_{i}$ correspond to the "matrix" transverse coordinates $X^{i}, i=1,2,3$ to the D-strings, which lie within D3-branes.

\subsection{Charge one monopoles}

In the case $k=1$ the analysis simplifies: $T_{i}=0$, and

$$
\Psi=\left(\begin{array}{c}
\left(\partial_{z}+x_{3}\right) v \\
\left(x_{1}+i x_{2}\right) v
\end{array}\right), \quad \partial_{z}^{2} v=r^{2} v, \quad r^{2}=\sum_{i} x_{i}^{2}
$$

The condition that $\Psi$ is finite at both ends of the interval allows two solutions for (4.27):

$$
v=e^{ \pm r z}
$$

which after imposing the normalization condition,(4.25), leads to:

$$
\Psi=\frac{1}{\sqrt{2 \sinh (r a)}}\left(\begin{array}{cc}
\sqrt{r+x_{3}} e^{r z} & -\sqrt{r-x_{3}} e^{-r z} \\
\frac{x_{+}}{\sqrt{r+x_{3}}} e^{r z} & \frac{x_{-}}{\sqrt{r-x_{3}}} e^{-r z}
\end{array}\right),
$$

where we used $x_{ \pm}=x_{1} \pm i x_{2}$.

In particular,

$$
\Phi=\frac{1}{2}\left(\frac{a}{\tanh (r a)}-\frac{1}{r}\right) \sigma_{3}
$$

\subsection{Abelian ordinary monopoles}

It is interesting that Nahm's equations describe Dirac monopoles as well. To achieve this replace the interval $(-a / 2, a / 2)$ by the interval $(-\infty, a)$. Intuitively this is natural, since in the $U(1)$ case the Higgs field $\phi$ has only one eigenvalue at infinity.

Then the equation (4.18) becomes simply the condition that the abelian monopole has a magnetic potential $\phi$, which must be harmonic. Let us find this harmonic function. The matrices $T_{i}$ can be taken to have the following form:

$$
T_{i}(z)=\frac{t_{i}}{z}, \quad\left[t_{i}, t_{j}\right]=i \varepsilon_{i j k} t_{k}
$$


where $t_{i}$ form an irreducible spin $j$ representation of $S U(2)$. Let $V \approx \mathbb{C}^{N}, N=2 j+1$, be the space of this representation. The matrices $\Psi_{ \pm}$are now $V$-valued. By an $S U(2)$ rotation we can bring the three-vector $x_{i}$ to the form $(0,0, r)$, i.e. $x_{1}=x_{2}=0, x_{3}>0$. Then one can show that in this basis

$$
\Psi_{-}=0, \quad \Psi_{+}=\nu_{j} z^{j} e^{r z}|j\rangle
$$

where $|j\rangle \in V$ is the highest spin state in $V$. The coefficient $\nu_{j}$ is found from the normalization condition:

$$
\left|\nu_{j}\right|^{2}=\frac{r^{2 j+1}}{(2 j) !} .
$$

From this we get the familiar formula for the singular Higgs field

$$
\phi=a-\frac{N}{2 r}
$$

\section{Abelian noncommutative monopoles}

In this section we study the solutions to the Bogomolny equations for U(1) gauge theory on a noncommutative three dimensional space. As before, we assume the Poisson structure $(\theta)$ which deforms the multiplication of the functions to be constant. Then there is essentially a unique choice of coordinate functions $x_{1}, x_{2}, x_{3}$ such that the commutation relations between them are as follows:

$$
\begin{aligned}
& {\left[x_{1}, x_{2}\right]=-i \theta, \quad \theta>0} \\
& {\left[x_{1}, x_{3}\right]=\left[x_{2}, x_{3}\right]=0}
\end{aligned}
$$

This algebra, (5.1), defines noncommutative $\mathbf{R}^{3}$, which we denote by $\mathcal{A}_{\theta}$. Introduce the creation and annihilation operators $c, c^{\dagger}$ :

$$
c=\frac{1}{\sqrt{2 \theta}}\left(x_{1}-i x_{2}\right), \quad c^{\dagger}=\frac{1}{\sqrt{2 \theta}}\left(x_{1}+i x_{2}\right)
$$

that obey

$$
\left[c, c^{\dagger}\right]=1
$$




\subsection{Noncommutative Nahm equations}

We start by repeating the procedure [10] that worked in the ADHM instanton case, namely we relax the condition that $x_{i}$ 's commute but insist on the equation (4.22) with $T_{i}$ replaced by the relevant matrices $\mathcal{T}_{i}=T_{i}+x_{i}$. Then the equation (4.22) on $T_{i}$ is modified:

$$
\partial_{z} T_{i}=i \varepsilon_{i j k} T_{j} T_{k}+\delta_{i 3} \theta
$$

It is obvious that, given a solution $T_{i}(z)$ of the original Nahm equations, it is easy to produce a solution of the noncommutative ones:

$$
T_{i}(z)^{\mathrm{nc}}=T_{i}(z)+\theta z \delta_{i 3}
$$

From this it follows that, unlike the case of instanton moduli space, the monopole moduli space does not change under noncommutative deformation.

This deformation, 23 (5.3), is exactly what one gets by looking at the D-strings suspended between the D3-branes (or a semi-infinite D-string with one end on a D3-brane) in the presence of a $B$-field. One gets the deformation:

$$
\left[X^{i}, X^{j}\right] \rightarrow\left[X^{i}, X^{j}\right]-i \theta^{i j}=\left[T_{i}, T_{j}\right]-\frac{1}{2} \theta \varepsilon_{i j 3}
$$

The reason why $\theta^{i j}$, instead of $B_{i j}$, appears on the right hand side of (5.5) is rather simple. By applying T-duality in the directions $x_{1}, x_{2}, x_{3}$ we could map the D-string into the D4brane. The matrices $X^{1}, X^{2}, X^{3}$ become the components $A_{\hat{1}}, A_{\hat{2}}, A_{\hat{3}}$ of the gauge field on the D4-brane worldvolume, and the $B$-field would couple to these gauge fields via the

standard coupling $F_{\hat{i} \hat{j}}-\hat{B}_{\hat{i} \hat{j}}$, where $\hat{B}_{\hat{i} \hat{j}}$ is the T-dualized $B$-field. It remains to observe that $\hat{B}_{\hat{i} \hat{j}}=\theta^{i j}$, since the T-dualized indices $\hat{i}$ label the coordinates on the space, dual to that of $x_{i}$ 's.

\subsection{Solving Nahm's equations}

To solve (5.3) we imitate the approach for the $k=1$ commutative monopole by taking

$$
T_{1,2}=0, T_{3}=\theta z
$$

To solve (4.24) for $\Psi$ we introduce the operators $b, b^{\dagger}$ :

$$
b=\frac{1}{\sqrt{2 \theta}}\left(\partial_{z}+x_{3}+\theta z\right), \quad b^{\dagger}=\frac{1}{\sqrt{2 \theta}}\left(-\partial_{z}+x_{3}+\theta z\right),
$$


which obey the oscillator commutation relations:

$$
\left[b, b^{\dagger}\right]=\left[c, c^{\dagger}\right]=1
$$

We introduce the superpotential

$$
W=x_{3} z+\frac{1}{2} \theta z^{2}
$$

so that $b=\frac{1}{\sqrt{2 \theta}} e^{-W} \partial_{z} e^{W}, \quad b^{\dagger}=-\frac{1}{\sqrt{2 \theta}} e^{W} \partial_{z} e^{-W}$. Then equation (4.24) becomes:

$$
\begin{aligned}
& b^{\dagger} \Psi_{+}+c \Psi_{-}=0 \\
& c^{\dagger} \Psi_{+}-b \Psi_{-}=0 .
\end{aligned}
$$

The general solution of (4.24) is:

$$
\Psi_{+}=\left(u_{1} b+u_{2} c\right) v, \quad \Psi_{-}=\left(u_{1} c^{\dagger}-u_{2} b^{\dagger}\right) v
$$

where $v$ satisfies

$$
\left(b^{\dagger} b+c c^{\dagger}\right) v=0
$$

and $\left(u_{1}, u_{2}\right)$ are two complex numbers defined up to multiplication by a common factor (which can be reabsorbed in the definition of $v$ ). Thus, the generic solution is parameterized by a point $u=\left(u_{1}: u_{2}\right) \in \mathbf{C P}^{1}$ on a two-dimensional (twistor) sphere.

To construct the solution we must now solve (5.12) and normalize $\Psi^{\dagger} \Psi$. Recall that $z$ is defined on the half line $(-\infty, a)$, so therefore $b$ and $b^{\dagger}$ are not Hermitian conjugates:

$$
\int \psi_{1}^{*} b \psi_{2}=\psi_{1}^{*} \psi_{2}(z=0)+\int\left(b^{\dagger} \psi_{1}\right)^{*} \psi_{2}
$$

As far as the $c, c^{\dagger}$ system is concerned we will work in the occupation number basis of the Fock space obtained by quantizing the $\left(x_{1}, x_{2}\right)$ plane. The most general expression for $v$ is:

$$
v=\sum_{n=0}^{\infty} v_{n m}\left(z, x_{3}\right)|n\rangle\langle m| .
$$

However it turns out that when $u=(1: 0)$ or when $u=(0: 1)$ one can make the following ansatz (which is equivalent to imposing axial symmetry on $v$ ):

$$
v=\sum_{n=0}^{\infty} v_{n}\left(z, x_{3}\right)|n\rangle\left\langle n\left|, \quad c c^{\dagger}\right| n\right\rangle=(n+1)|n\rangle .
$$


In this case (5.12) becomes

$$
b^{\dagger} b v_{n}\left(z, x_{3}\right)=-(n+1) v_{n}\left(z, x_{3}\right),
$$

in the class of functions that lead to a $\Psi$ that is normalizable on the half line $(-\infty, a)$. It is obvious that if we can solve for $v_{0}$ then $v_{n} \propto b^{n} v_{0}$; so the solution of (5.12) is

$$
v_{n}=\nu_{n} b^{n} \varphi(z)
$$

where

$$
\varphi(z) \equiv e^{W(z)} \int_{-\infty}^{z} e^{-2 W(p)} d p
$$

and $\nu_{n}$ are normalization constants to be determined below. Will this lead to a normalizable $\Psi$ ? As $z \rightarrow-\infty$ we have the estimate $\varphi(z)<e^{-W(z)}$, so that $v_{0}$, as well as all its descendants $v_{n}$, are good functions. Notice that we can only make it normalizable on a half line, which nicely fits with the intuition that the abelian Higgs field must have values that are bounded.

- Notice that by shifting the coordinate $x_{3} \mapsto x_{3}+\theta a$ we can always make $a=0$ (this is impossible for $\theta=0$ ). From now on we assume $a=0$.

- After this shift we see that the only dimensional parameter in the problem is $\theta$. Let us choose the length units in which $2 \theta=1$.

In the case, where $u$ is a generic point on the two sphere, we have

$$
v=\sum_{n, m} \nu_{n m} b^{n} \varphi(z)|n\rangle\langle m|
$$

In this paper we shall only discuss the case where either $u=(1: 0)$, or $u=(0: 1)$.

\subsection{The normalization condition}

We start by considering the case $u=(0: 1)$. Accordingly, $\Psi_{+}=c v, \quad \Psi_{-}=-b^{\dagger} v$, and

$$
\Psi^{\dagger} \Psi=\sum_{n=0}^{\infty}\left[\left(b^{\dagger} v_{n}\right)^{\dagger}\left(b^{\dagger} v_{n}\right)+n\left|v_{n}\right|^{2}\right]|n\rangle\left\langle n\left|=-\sum_{n=0}^{\infty} \partial_{z}\left(v_{n}^{\dagger} b^{\dagger} v_{n}\right)\right| n\right\rangle\langle n| .
$$

The noncommutative version of the condition (4.25) is:

$$
\int_{-\infty}^{0} d z \Psi^{\dagger} \Psi=1=\sum_{n=0}^{\infty}|n\rangle\langle n|
$$


thus

$$
\left(v_{n}^{\dagger} b^{\dagger} v_{n}\right)(z=0)=-1 .
$$

which reduces to the sequence of relations:

$$
n\left|\nu_{n}\right|^{2}\left(\partial^{n} \Upsilon\left(2 x_{3}\right)\right)\left(\partial^{n-1} \Upsilon\left(2 x_{3}\right)\right)=1
$$

where

$$
\begin{aligned}
\Upsilon(z)= & \int_{0}^{\infty} e^{-\frac{p^{2}}{2}+z p} d p \\
& =\sqrt{\frac{\pi}{2}} e^{\frac{z^{2}}{2}}\left(1+\operatorname{erf}\left(\frac{z}{\sqrt{2}}\right)\right) \\
& =\sum_{n=0}^{\infty} \frac{\left(\frac{n-1}{2}\right) !}{n !} 2^{\frac{n-1}{2}} z^{n} \\
& \sim \sqrt{2 \pi} e^{\frac{z^{2}}{2}}, \quad z \rightarrow+\infty \\
& \sim \frac{1}{|z|}, \quad z \rightarrow-\infty .
\end{aligned}
$$

For $n=0$ (5.21) is explicitly given by the analytic continuation of (5.21) to $n=0$, namely $\left|\nu_{0}\right|^{2} \Upsilon\left(2 x_{3}\right)=1$. The function (5.22) obeys the following differential equation

$$
\partial_{z} \Upsilon(z)=z \Upsilon(z)+1, \quad \Upsilon(0)=\sqrt{\frac{\pi}{2}} .
$$

Introduce the expansion coefficients

$$
\begin{aligned}
\Upsilon\left(2 x_{3}+y\right) & =\sum_{n=0} \zeta_{n} \frac{y^{n}}{n !}, \\
\zeta_{n} & =\int_{0}^{\infty} p^{n} e^{-\frac{p^{2}}{2}+2 p x_{3}} d p
\end{aligned}
$$

which obey the following equations:

$$
\begin{aligned}
\zeta_{n+1} & =2 x_{3} \zeta_{n}+n \zeta_{n-1} \\
\partial_{3} \zeta_{n} & =2 \zeta_{n+1} \\
\zeta_{n}\left(x_{3}=0\right) & =2^{\frac{n-1}{2}}\left(\frac{n-1}{2}\right) ! .
\end{aligned}
$$

The recursion relation in (5.25) for $n=0$ is to be understood by analytic continuation as $n \rightarrow 0$. In this limit we have $n \zeta_{n-1} \rightarrow 1$, as $n \rightarrow 0$. Thus $\zeta_{1}=2 x_{3} \zeta_{0}+1$, as can also be checked directly from (5.24). 
To find the normalization constants we substitute (5.24) into (5.21) to deduce:

$$
\left|\nu_{n}\right|^{2}=\frac{1}{n \zeta_{n} \zeta_{n-1}} .
$$

Again, for $n=0$ the last equality is understood as $\left|\nu_{0}\right|^{2}=1 / \zeta_{0}$. This completes the solution. We have explicitly constructed $v$ and thus $\Psi_{ \pm}$, from which we can determine, using (4.26), the Higgs and gauge fields. To do this we shall need to evaluate the overlap integrals:

$$
\int_{-\infty}^{0}\left(b^{n} \varphi\right)\left(b^{n+1} \varphi\right)=\zeta_{n+2} \zeta_{n}-\zeta_{n+1}^{2}=(n+1) \zeta_{n}^{2}-n \zeta_{n+1} \zeta_{n-1} .
$$

Again, for $n=0$ the last equality is understood with $n \zeta_{n-1}=1$ for $n=0$.

Let us also introduce the functions $\xi, \tilde{\xi}$ and $\eta=\tilde{\xi}^{2}$ :

$$
\tilde{\xi}(n)=\sqrt{\frac{\zeta_{n}}{\zeta_{n+1}}}, \quad \eta(n)=\frac{\zeta_{n}}{\zeta_{n+1}}, \quad \xi(n)=\sqrt{\frac{n \zeta_{n-1}}{\zeta_{n}}} .
$$

We will need the asymptotics of these functions for large $x_{3}$. Let $r_{n}^{2}=x_{3}^{2}+n$. For $r_{n}+x_{3} \rightarrow \infty$ we can estimate the integral in (5.24) by the saddle point method. The saddle point and the approximate values of $\zeta_{n}$ and $\eta_{n}$ are:

$$
\begin{aligned}
& \bar{p}=x_{3}+r_{n} \\
& \zeta_{n} \sim \sqrt{\frac{\pi}{r_{n}}}\left(x_{3}+r_{n}\right)^{n+\frac{1}{2}} e^{\frac{1}{2}\left(x_{3}+r_{n}\right)\left(3 x_{3}-r_{n}\right)} \\
& \eta_{n} \sim \frac{1}{x_{3}+r_{n+1}}\left(1+\frac{1}{4 r_{n}^{2}}+\ldots\right) .
\end{aligned}
$$

\subsection{The explicit solution for the gauge and Higgs fields}

The Higgs Field.

The Higgs field is given by (4.26):

$$
\Phi=\int d z z \Psi^{\dagger} \Psi \equiv \sum_{n=0}^{\infty} \Phi_{n}\left(x_{3}\right)|n\rangle\langle n|,
$$

it has axial symmetry, that is commutes with the the number operator $c^{\dagger} c$. Explicitly:

$$
\begin{aligned}
\Phi_{n}= & \int v_{n}^{*} b^{\dagger} v_{n} d z= \\
& =\frac{\zeta_{n}}{\zeta_{n-1}}-\frac{\zeta_{n+1}}{\zeta_{n}}=\partial_{3} \log \xi_{n} \\
= & (n-1) \eta_{n-2}-n \eta_{n-1}, \quad n>0 \\
= & -\frac{\zeta_{1}}{\zeta_{0}}=-2 x_{3}-\frac{1}{\zeta_{0}}, \quad n=0 .
\end{aligned}
$$


To arrive at the third line we used the fact that

$$
\frac{1}{\eta_{n}}-\frac{1}{\eta_{n+1}}=n \eta_{n-1}-(n+1) \eta_{n},
$$

which follows immediately from the recursion relation for the $\zeta^{\prime} \mathrm{s}$ in (5.25). These fields are finite at $x_{3}=0$. Indeed as $x_{3} \rightarrow 0$,

$$
\Phi_{n}\left(x_{3}=0\right) \quad=\sqrt{2}\left(\frac{\left(\frac{n-1}{2}\right) !}{\left(\frac{n-2}{2}\right) !}-\frac{\left(\frac{n}{2}\right) !}{\left(\frac{n-1}{2}\right) !}\right) .
$$

At the origin:

$$
\Phi_{0}\left(x_{3}=0\right)=-\sqrt{\frac{2}{\pi}} .
$$

The Gauge Field.

Using (4.26) it is easy to see that the component $A_{3}$ vanishes

$$
\begin{aligned}
A_{3}= & \int\left(b^{\dagger} v_{n}\right)^{\dagger} \partial_{3}\left(b^{\dagger} v_{n}\right)+n v_{n}^{\dagger} \partial_{3} v_{n}= \\
& -\left(b^{\dagger} v_{n}\right)^{\dagger} \partial_{3} v_{n}(z=0)+\int\left(b^{\dagger} v_{n}\right)^{\dagger} v_{n}= \\
& \partial_{3} \log \xi(n)+\frac{\zeta_{n+1} \zeta_{n-1}-\zeta_{n}^{2}}{\zeta_{n-1} \zeta_{n}}=0 .
\end{aligned}
$$

In the same gauge the components $A_{1}, A_{2}$ (which we consider to be anti-hermitian) are given by:

$$
\begin{aligned}
& A_{c}=\frac{1}{2}\left(A_{1}+i A_{2}\right), A_{c^{\dagger}}=\frac{1}{2}\left(A_{1}-i A_{2}\right)=-A_{c}^{\dagger} \\
& A_{c}=\int \Psi^{\dagger}\left[\Psi, c^{\dagger}\right] \\
&=\xi^{-1}\left[\xi, c^{\dagger}\right]=c^{\dagger}\left(1-\frac{\xi(n)}{\xi(n+1)}\right) .
\end{aligned}
$$

Again we see that the matrix elements of $A_{c}$ are all finite and non singular.

The Field strength.

From (5.35) we deduce:

$$
\begin{gathered}
F_{12}=2 i\left(\partial_{c} A_{c^{\dagger}}-\partial_{c^{\dagger}} A_{c}+\left[A_{c}, A_{c^{\dagger}}\right]\right)= \\
2\left(\left[\frac{\xi(n)}{\xi(n+1)} c, c^{\dagger} \frac{\xi(n)}{\xi(n+1)}\right]-1\right)= \\
=2 \sum_{n>0}\left(-1+(n+1)\left(\frac{\xi(n)}{\xi(n+1)}\right)^{2}-n\left(\frac{\xi(n-1)}{\xi(n)}\right)^{2}\right)|n\rangle\langle n|+ \\
+2\left(-1+\left(\frac{\xi(0)}{\xi(1)}\right)^{2}\right)|0\rangle\langle 0|
\end{gathered}
$$


from which it follows, that:

$$
\begin{aligned}
B_{3}(n) & =2\left(1-n \frac{\eta_{n-1}}{\eta_{n}}+(n-1) \frac{\eta_{n-2}}{\eta_{n-1}}\right) \\
B_{c} & =\frac{1}{2}\left(B_{1}+i B_{2}\right)=c^{\dagger} \frac{\xi(n)}{\xi(n+1)}(\Phi(n)-\Phi(n+1)) .
\end{aligned}
$$

with the understanding that at $n=0$ :

$$
B_{3}(0)=2\left(1-\frac{\zeta_{1}}{\zeta_{0}^{2}}\right) .
$$

\subsection{Checking the Bogomolny equations.}

With our conventions it is relatively easy to check that our solution satisfies the Bogomolny equations everywhere:

$$
\begin{aligned}
& \nabla_{3} \Phi=\partial_{3} \Phi=-B_{3} \\
& \nabla_{c} \Phi=\xi^{-1} \partial_{c} \Phi \xi=-B_{c} .
\end{aligned}
$$

For example, consider the equation $\partial_{3} \Phi=-B_{3}$. We have:

$$
\partial_{3} \Phi(n)=\partial_{3}\left[(n-1) \eta_{n-2}-n \eta_{n-1}\right] .
$$

Then we use the fact that

$$
\partial_{3} \eta_{n}=2\left(1-\frac{\eta_{n}}{\eta_{n+1}}\right),
$$

to see that

$$
\begin{aligned}
& \partial_{3} \Phi(n)=2(n-1)\left(1-\frac{\eta_{n-2}}{\eta_{n-1}}\right)-2 n\left(1-\frac{\eta_{n-1}}{\eta_{n}}\right)= \\
& -2\left(1-n \frac{\eta_{n-1}}{\eta_{n}}+(n-1) \frac{\eta_{n-2}}{\eta_{n-1}}\right)=-B_{3}(n) .
\end{aligned}
$$

Other solutions and Seiberg-Witten map.

It is plausible that the solutions corresponding to the other values of $u=\left(u_{1}: u_{2}\right)$ also have a physical meaning. In fact, the solutions of the Dirac-Born-Infeld theory [24] [25] [13] that describe a a D-string touching a D3-brane (or D-string suspended between two D3-branes) in the presence of the $B$-field suggest that $\Phi$ is multi-valued. Moreover, the solution for $\Phi$ [24] is implicit, whereas our solution is explicit. On the other hand the Seiberg-Witten map from the noncommutative gauge fields to the commutative ones [5] must map our explicit solution for $(A, \Phi)$ into the solution of the DBI theory. It could mean that our solution is just one branch of the full solution, somehow incorporating other choices of $u$. 
However, we have found that the solution corresponding to the choice $u=(1: 0)$ does not quite satisfy the BPS equations everywhere. Instead, it has a source, localized along a semi-infinite string pointing in the $x_{3} \rightarrow-\infty$ direction. Nevertheless, it is clear that the other "branches", corresponding to the generic $u$, seem worth investigating further. It is also plausible that in order to have a better understanding of the matching of the solutions to the DBI theory and the noncommutative gauge theory one would need to incorporate the $\alpha^{\prime}$ corrections.

A remark concerning instantons.

As in the ordinary gauge theory case the monopoles are the solutions of the instanton equations in four dimensions, that are invariant under translations in the fourth direction $x_{4}$. We observe that the solution presented above $((5.31),(5.35))$, can also be cast in the Yang form: Take $\xi=\xi\left(x_{3}, n\right)$ as in (5.35). Then $\partial_{3} \xi$ commutes with $\xi$ and we can write $\partial_{3} \xi \xi^{-1}=\partial_{3} \log \xi$. The formulae (4.7) yield exactly (5.35) and (5.31) with $\Phi=i A_{4}$. Indeed, the equation (4.8) is nothing but the first equation in (5.39).

\subsection{Toda lattice}

At this point it is worth mentioning the relation of the noncommutative Bogomolny equations with the Polyakov's non-abelian Toda system (see [26] for the recent studies of this system) on the semi-infinite one-dimensional lattice. Let us try to solve the equations (4.18) using the Yang ansatz and imposing the axial symmetry: we assume that $\xi\left(x_{1}, x_{2}, x_{3}\right)=\xi\left(n, x_{3}\right), n=c^{\dagger} c$. Then the equation (4.8) for the $x_{4}$-independent fields reduces to the system:

$$
\partial_{t}\left(\partial_{t} g_{n} g_{n}^{-1}\right)-g_{n} g_{n+1}^{-1}+g_{n-1} g_{n}^{-1}=0
$$

where

$$
g_{n}(t)=\frac{e^{\frac{t^{2}}{2}}}{n !} \xi^{2}\left(n, \frac{t}{2}\right),
$$

(notice that $g_{n}(t)$ are ordinary matrices). In the $U(1)$ case we can write

$$
g_{n}(t)=e^{\alpha_{n}(t)}
$$

and rewrite (5.42) in a more familiar form:

$$
\partial_{t}^{2} \alpha_{n}+e^{\alpha_{n-1}-\alpha_{n}}-e^{\alpha_{n}-\alpha_{n+1}}=0
$$


For $n=0$ these equations also formally hold if we set $g_{-1}=0$ (this boundary condition follows both from the Bogomolny equations and the same condition is imposed on the Toda variables on the lattice with the end-points).

Our Higgs field $\Phi_{n}$ has a simple relation to the $\alpha$ 's:

$$
\Phi\left(x_{3}, n\right)=-2 x_{3}+\alpha_{n}^{\prime}\left(2 x_{3}\right)
$$

Our solution to 5.43 is:

$$
\alpha_{n}=\frac{1}{2} t^{2}+\log \left(\frac{n \zeta_{n-1}(t / 2)}{\zeta_{n}(t / 2)}\right)-\log (n !) .
$$

It is amusing that Polyakov's motivation for studying the system (5.42) was the structure of loop equations for lattice gauge theory. Here we encountered these equations in the study of the continuous, but noncommutative, gauge theory, thus giving more evidence for their similarity.

We should note in passing that in the integrable non-abelian Toda system one usually has two 'times' $t, \bar{t}$, so that the equation (5.42) has actually the form [26]:

$$
\partial_{t}\left(\partial_{\bar{t}} g_{n} g_{n}^{-1}\right)-g_{n} g_{n+1}^{-1}+g_{n-1} g_{n}^{-1}=0
$$

It is obvious that these equations describe four-dimensional axial symmetric instantons on the noncommutative space with the coordinates $t, \bar{t}, c, c^{\dagger}$ of which only half is noncommuting.

\subsection{The mass of the monopole}

In this section we restore our original units, so that $2 \theta$ has dimensions of (length) ${ }^{2}$. From the formulae 5.29 ) we can derive the following estimates:

$$
\Phi(n) \sim-\frac{1}{2 r_{n}}=-\frac{1}{2 \sqrt{x_{3}^{2}+2 \theta n}} \quad n \neq 0, r \rightarrow \infty .
$$

Instead, for $n=0$ we have:

$$
\begin{aligned}
& \Phi(0) \sim-\frac{x_{3}}{\theta}, \quad x_{3} \rightarrow+\infty \\
& \Phi(0) \sim-\frac{1}{2\left|x_{3}\right|}, \quad x_{3} \rightarrow-\infty .
\end{aligned}
$$


The asymptotics of the magnetic field is clear from the Bogomolny equations and the behaviour of $\Phi$. Thus, for example,

$$
B_{3}(n)=-\partial_{3} \Phi(n)=-\frac{x_{3}}{2 r_{n}^{3}}, \quad n \neq 0
$$

and similarly for the other components of $B$. This is easily translated into ordinary position space, as in the discussion following equation(3.21), since, for large $n, B_{i}\left(n, x_{3}\right) \sim B_{i}\left(x_{1}^{2}+\right.$ $\left.x_{2}^{2} \sim n, x_{3}\right)$. Therefore the magnetic field for large values of $x_{3}$ and $n$, or equivalently large $x_{i}$ is that of a point-like magnetic charge at the origin. However the $n=0$ component of $B_{3}$ behaves differently for large positive $x_{3}$ :

$$
B_{3}(n=0)=-\partial_{3} \Phi(0)=\frac{1}{\theta} .
$$

Notice, that this is exactly the value of the $B$-field. Thus, in addition to the magnetic charge at the origin we have a flux tube, localized in a Gaussian packet in the $\left(x_{1}, x_{2}\right)$ plane, of the size $\propto \theta$, along the positive $x_{3}$ axis. The monopole solution is indeed a smeared version of the Dirac monopole, wherein the Dirac string (the D-string!) is physical.

To calculate the energy of the monopole we use the Bogomolny equations to reduce the total energy to a boundary term:

$$
\begin{aligned}
\mathcal{E}= & \frac{1}{2 g_{\mathrm{YM}}^{2}} \int d^{3} x(\vec{B} \star \vec{B}+\vec{\nabla} \Phi \star \vec{\nabla} \Phi)= \\
& \frac{1}{2 g_{\mathrm{YM}}^{2}} \int d^{3} x(\vec{B}+\vec{\nabla} \Phi)^{2}-\frac{1}{2 g_{\mathrm{YM}}^{2}} \int d^{3} x \vec{\nabla} \cdot(\vec{B} \star \Phi+\Phi \star \vec{B})= \\
& \frac{2 \pi \theta}{2 g_{\mathrm{YM}}^{2}} \int d x_{3} \sum_{n}\left\langle n\left|\partial_{3}^{2} \Phi^{2}+4 \partial_{c}\left(\xi^{2}\left(\partial_{c^{\dagger}} \Phi^{2}\right) \xi^{-2}\right)\right| n\right\rangle,
\end{aligned}
$$

where in the last line we switched back to the Fock space, by using the relation:

$$
\int d x_{1} d x_{2} f\left(x_{1}, x_{2}\right)=2 \pi \theta \operatorname{Tr}_{\mathcal{H}} \hat{f}
$$

Thus, the energy is given by the boundary term. To evaluate this expression we need to figure out what the boundary term is in the noncommutative, Fock space setup?

Consider the derivative terms in (5.50) involving $x_{1}, x_{2}$. They can be expressed as the commutators with $c$ or $c^{\dagger}$. In computing the trace

$$
\operatorname{Tr}_{\mathcal{H}}[c, \mathcal{X}]=\sum_{n}\langle n|[c, \mathcal{X}]| n\rangle
$$


where we denote by $\mathcal{X}$ the terms $\Phi B_{c}+B_{c} \Phi$ in $(5.50)$, we get naively get that the trace of a commutator is zero. But we should be careful, since the matrices are infinite and the trace is an infinite sum. If we regulate it by restricting the sum to $n \leq N$, then the matrix element $\langle N|c| N+1\rangle\langle N+1|\mathcal{X}| N\rangle$ is not cancelled, so that the regularized trace is

$$
\operatorname{Tr}_{\mathcal{H}}[c, \mathcal{X}]=\sqrt{N+1}\langle N+1|\mathcal{X}| N\rangle
$$

and similarly for $c^{\dagger}$. Let us choose as the infrared regulator box the "region" where $\left|x_{3}\right| \leq L, 0 \leq n \leq N, L \sim \sqrt{2 \theta N} \gg 1$. Then the total integral in (5.50) reduces to the sum of two terms (up to the factor $\frac{\pi \theta}{g_{\mathrm{YM}}^{2}}$ ):

$$
\begin{gathered}
4 N \int_{-L}^{L} d x_{3} \frac{\eta_{N-1}}{\eta_{N}}\left(\Phi_{N}^{2}-\Phi_{N+1}^{2}\right) \\
+\left.\sum_{n=0}^{N} \partial_{3} \Phi_{n}^{2}\right|_{x_{3}=-L} ^{x_{3}=+L} .
\end{gathered}
$$

The first line in (5.54) is easy to evaluate. Since $x_{3}+r_{n} \geq-L+\sqrt{L^{2}+2 \theta N} \propto \sqrt{\theta N} \rightarrow \infty$ we can use the asymptotic expressions (5.29) to make an estimate:

$$
\begin{gathered}
4 N \int_{-L}^{L} d x_{3} \frac{\zeta_{N+1} \zeta_{N-1}}{\zeta_{N}^{2}}\left(\Phi_{N}^{2}-\Phi_{N+1}^{2}\right) \approx \int_{-L}^{L} d x \frac{2 \theta N}{\left(x^{2}+2 \theta N\right)^{2}} \\
\approx \frac{L}{L^{2}+2 \theta N} \rightarrow 0
\end{gathered}
$$

The second line in (5.54) contains derivatives of the Higgs field evaluated at $x_{3}=L \gg 0$ and at $x_{3}=-L \ll 0$. The former is estimated using the $z \gg 0$ asymptotics in (5.22) or (5.29), and produces:

$$
\sum_{n=0}^{N} \partial_{3} \Phi_{n}^{2}\left(x_{3}=L\right) \sim \frac{2 \theta(N-1)}{L^{3}}+2 \frac{L}{\theta^{2}}
$$

The diverging with $L$ piece comes solely from the $n=0$ term. Finally, the $x_{3}=-L$ case is treated via $z \ll 0$ asymptotics in (5.22) yielding the estimate $\sim \theta N / L^{3}$ vanishing in the limit of large $L, N$.

Hence the total energy is given by

$$
\mathcal{E} \propto \frac{2 \pi \theta \times 2 L}{2 g_{\mathrm{YM}}^{2} \theta^{2}}=\frac{2 \pi L}{g_{\mathrm{YM}}^{2} \theta},
$$


which is the mass of a string of length $L$ whose tension is

$$
T=\frac{2 \pi}{g_{\mathrm{YM}}^{2} \theta} .
$$

\subsection{Magnetic charge}

It is instructive to see what is the magnetic charge of our solution. On the one hand, it is clearly zero:

$$
Q \propto \int_{\partial(\text { space })} \vec{B} \cdot d \vec{S}=\int d^{3} x \vec{\nabla} \cdot \vec{B}=0
$$

since the gauge field is everywhere non-singular. On the other hand, we were performing a $\theta$-deformation of the Dirac monopole, which definitely had magnetic charge. To see what has happened let us look at (5.56) more carefully. We again introduce the box and evaluate the boundary integral (5.56) as in (5.53):

$$
\frac{Q}{2 \pi}=\sum_{n=0}^{N}\left[B_{3}\left(x_{3}=L, n\right)-B_{3}\left(x_{3}=-L, n\right)\right]+4 N \int_{-L}^{L} d x_{3} \frac{\eta_{N-1}}{\eta_{N}}\left(\Phi_{N}-\Phi_{N+1}\right)
$$

It is easy to compute the sums

$$
\begin{gathered}
\sum_{n=0}^{N} B_{3}(x, n)=\partial_{3} \frac{\zeta_{N+1}}{\zeta_{N}} \\
4 N \int_{-L}^{L} d x_{3} \frac{\eta_{N-1}}{\eta_{N}}\left(\Phi_{N}-\Phi_{N+1}\right)=4(N+1) \int \frac{\xi_{N}^{2}}{\xi_{N+1}^{2}} d \log \frac{\xi_{N}}{\xi_{N+1}}= \\
=\left.2(N+1)\left(\frac{\xi_{N}}{\xi_{N+1}}\right)^{2}\right|_{\left.\right|_{3}=-L} ^{x_{3}=+L}=\left.2 N \frac{\zeta_{N-1} \zeta_{N+1}}{\zeta_{N}^{2}}\right|_{x_{3}=-L} ^{x_{3}=+L},
\end{gathered}
$$

and the total charge vanishes as:

$$
Q=\left.\left[2 N \frac{\zeta_{N-1} \zeta_{N+1}}{\zeta_{N}^{2}}+\partial_{3}\left(\frac{\zeta_{N+1}}{\zeta_{N}}\right)\right]_{x_{3}=-L}^{x_{3}=+L} \equiv 2(N+1)\right|_{x_{3}=+L}-\left.2(N+1)\right|_{x_{3}=-L}
$$

We can better understand the distribution of the magnetic field by looking separately at the fluxes through the "lids" $x_{3}= \pm L$ of our box and through the "walls" $n=N$.

The walls contribute

$$
\left[2 N \frac{\zeta_{N-1} \zeta_{N+1}}{\zeta_{N}^{2}}\right]_{x_{3}=-L}^{x_{3}=+L} \sim-\frac{L}{\sqrt{L^{2}+N}} \sim-1
$$

while the lids contribute $\sim+1$. Let us isolate the term $B_{3}(+L, n=0) \rightarrow+2$ (recall (5.47)). It contributes to the flux through the upper lid. The rest of the flux through the 
lids is therefore $\sim-1$. Hence the flux through the rest of the "sphere at infinity" is -2 and it is roughly uniformly distributed ( -1 contribute the walls and -1 the lids). So we get a picture of a spherical magnetic field of a monopole together with a flux tube pointing in one direction.

This spherical flux becomes observable in the naive $\theta \rightarrow 0$ limit, in which the string becomes localized at the point $x_{3}=0, n=0$ (since the slope of the linearly growing $\Phi_{0} \sim \frac{x_{3}}{\theta}$ becomes infinite). In the $\theta=0$ limit we throw out this point and all of the string.

\section{Discussion}

In this paper we found an explicit analytic expression for a soliton in the $\mathrm{U}(1)$ gauge theory on a noncommutative space. The solution describes a magnetic monopole attached to a finite tension string, that runs off to infinity tranverse to the noncommutative plane. This soliton has a clear reflection in type IIB string theory. If the gauge theory is realized as the $\alpha^{\prime} \rightarrow 0$ limit of the theory on a D3-brane in the IIB string theory in the presence of a background NS B-field, then the monopole with the string attached is nothing but the D1-string ending on the D3-brane. What is unusual about the solution that we found is that it describes this string as a non-singular field configuration.

Whether this string is a dynamical object in the gauge theory, with full stringy degrees of freedom, remains to be determined. To this end we should analyze the spectrum of the fluctuations of this string. Several remarks are in order:

- First, the "location" of the string is not very well defined., In noncommutative gauge theory the local energy density, as all local operators, is not gauge invariant. However the energy of a line element of a string, as a function of $x_{3}$ is a well-defined gauge-invariant notion:

$$
t\left(x_{3}\right)=\frac{1}{2 g_{\mathrm{YM}}^{2}} \int d x_{1} d x_{2}\left(\vec{B}^{2}+(\vec{\nabla} \Phi)^{2}\right) .
$$

For our solution this "tension" turned out to be exponentially small for $x_{3}<0$ and essentially a constant

$$
t=\frac{2 \pi}{g_{\mathrm{YM}}^{2} \theta},
$$

for $x_{3}>0$.

- We worked in the gauge where $A_{3}=0$, which still allows for $x_{3}$-independent gauge transformations. This gauge freedom is broken down to a global $U(1)$ rotation by demanding that for $x_{3} \rightarrow+\infty$

$$
\Phi_{0}\left(x_{3}\right) \rightarrow-\frac{x_{3}}{\theta} .
$$


If we impose this asymptotic behaviour on $\Phi$, then the problem of finding the spectrum of the fluctuations of the string becomes well-posed.

- Our solution breaks translational invariance. One would expect the derivatives $\partial_{\mu}\left(\Phi, A_{c}, A_{c^{\dagger}}\right)$ to show up as zero modes. However, the derivatives in the $x_{1}, x_{2}$ directions are infinitesimal gauge transformations, while the derivatives in the $x_{3}$ direction are not normalizable:

$$
\partial_{c} \Phi=\left[\Phi, c^{\dagger}\right], \quad \partial_{c} A_{\mu}=D_{\mu} c^{\dagger}-\delta_{\mu, c^{\dagger}}
$$

(the shift of $A_{\mu}$ by a constant is a symmetry of the theory).

The next subject which we plan to elaborate further on is the extension of our analytic solution to the case of $U(2)$ noncommutative gauge theory. In this case one expects to find strings of finite extent, according to the brane picture [12].

What is the relation between the string we have found and the electric flux strings found recently in [15]. These authors also study the coupled gauge field - Higgs field system, with the Higgs field in the adjoint representation. Their Higgs field $t$ arises from the open string tachyon, and has a non-trivial potential $V(t)$. In the limit of large noncommutativity $\theta$ the kinetic term can be neglected, according to [27], and the soliton can be found as a Gaussian wave-packet localized at the origin of the transverse plane to the would-be-string space, with the values of the tachyon field at the origin and far away given by at two different critical points of the potential $V(t)$. In our case we have no potential for $\Phi$, nor did we assume $\theta$ to be large. However, our solitonic string also had an effective thickness of the order of $\theta$, and also disappears when $\theta=0$. It would be interesting to see, whether S-duality will map our magnetic strings to the electric strings of [15].

As a step in this direction we would like to compare the tension of our string with that of D-string (the authors of [15] claim to have a complete agreement of the tension of their soliton with the tension of the fundamental string). As already mentioned, a Dstring ending on a D3-brane in the presence of the constant $B$-field bends. To analyze this bending one could use the exact solution of the DBI theory [24], the B-deformed spike solutions of [28]. However, for our qualitative analysis, it is sufficient to look at the linearized equations. If we replace the DBI Lagrangian by its Maxwell approximation, then the BPS equations in the presence of the $B$-field will have the form:

$$
B_{i j}+F_{i j}+\sqrt{\operatorname{det} g} \varepsilon_{i j k} g^{k l} \partial_{l} \Phi=0,
$$


where we should use the closed string metric (4.12). The solution of $(6.3)$ is:

$$
\Phi=B\left(1+\left(\frac{\theta}{2 \pi \alpha^{\prime}}\right)^{2}\right) x_{3}-\frac{1}{2 r}, \quad r^{2}=x_{3}^{2}+\frac{1}{\left(1+\left(\frac{\theta}{2 \pi \alpha^{\prime}}\right)^{2}\right)}\left(x_{1}^{2}+x_{2}^{2}\right)
$$

The linearly growing piece in $\Phi$ should be interpreted as a global rotation of the D3-brane, by an angle $\psi, \tan \psi=\frac{\theta}{\left(2 \pi \alpha^{\prime}\right)}$. This conclusion remains correct even after the full non-linear BPS equation is solved (see [24]. Notice however that we fix $G_{i j}=\delta_{i j}$ instead of $g_{i j}=\delta_{i j}$ as in [24]). The singular part of $\Phi$, the spike, represents the D-string. If we rotate the brane, then the spike forms an angle $\frac{\pi}{2}-\psi$ with the brane. If we project this spike on the brane, then the energy, carried by its shadow per unit length, is related to the tension of the D-string via:

$$
\frac{T_{D 1}}{\sin \psi}=\frac{1}{2 \pi \alpha^{\prime} g_{s}} \frac{\sqrt{\left(2 \pi \alpha^{\prime}\right)^{2}+\theta^{2}}}{\theta}=\frac{\left(2 \pi \alpha^{\prime}\right)^{2}+\theta^{2}}{2 \pi g_{\mathrm{YM}}^{2}\left(\alpha^{\prime}\right)^{2} \theta} .
$$

However, this is not the full story. The endpoint of the D-string is a magnetic charge, which experiences a constant force, induced by the background magnetic field. If we had introduced a box of the extent $2 L$ in the $x_{3}$-direction, then in order to bring a tilted Dstring into our system from outside of the box we would have had to spend an energy equal to $\frac{T_{D 1}}{\sin \psi} L$, but we would have been helped by the magnetic force, which would decrease the work done by

$$
\frac{2 \pi}{g_{\mathrm{YM}}^{2}} B_{3}=\frac{2 \pi}{g_{\mathrm{YM}}^{2}} B_{12} g^{11} g^{22} \sqrt{g} \sim \frac{1}{\left(\alpha^{\prime} g_{\mathrm{YM}}\right)^{2}} \theta .
$$

In sum, the energy of the semi-infinite D-string in the box will be given byt:

$$
\frac{2 \pi}{g_{\mathrm{YM}}^{2} \theta} \text {. }
$$

This expression coincides with our tension (6.2). On dimensional grounds, noncommutative gauge theory cannot produce any other dependence of the tension on $\theta$ but that given in (6.2).

Finally, the large $\theta$ limit of the noncommutative gauge theory may provide an exciting opportunity to learn more about the large $N$ non-abelian commutative gauge theories, for both theories become essentially planar in this limit. If we keep $g_{\mathrm{YM}}^{2}$ small and take $\theta \rightarrow \infty$ then our magnetic strings become tensionless. Whether this could lead to condensation of the magnetic charges and a mechanism for confinement remains to be seen.

1 We thank K. Hashimoto for bringing a very helpful argument from the second reference in [12] to our attention 


\section{References}

[1] H. S. Snyder, "Quantized Space-Time", Phys. Rev. 71 (1947) 38; "The Electromagnetic Field in Quantized Space-Time", Phys. Rev. 72 (1947) 68

[2] A. Connes, "Noncommutative geometry", Academic Press (1994)

[3] A. Connes, M. Douglas, A. Schwarz, JHEP 9802(1998) 003

[4] V. Schomerus, "D-Branes and Deformation Quantization", JHEP 9906(1999) 030

[5] N. Seiberg, E. Witten, hep-th/9908142, JHEP 9909(1999) 032

[6] T. Filk, "Divergencies in a Field Theory on Quantum Space", Phys. Lett. 376B (1996) 53

[7] S. Minwala, M. van Raamsdonk, N. Seiberg, "Noncommutative Perturbative Dynamics", hep-th/9912072

[8] E. Witten, Nucl. Phys. B268 (1986) 253

[9] M. van Raamsdonk, N. Seiberg, "Comments of Noncommutative Perturbative Dynamics", hep-th/0002186, JHEP 0003(2000) 035

[10] N. Nekrasov, A. S. Schwarz, hep-th/9802068, Comm. Math. Phys. 198 (1998) 689

[11] H. Braden, N. Nekrasov, hep-th/9912019;

K. Furuuchi, hep-th/9912047

[12] K. Hashimoto, H. Hata, S. Moriyama, hep-th/9910196, JHEP 9912(1999) 021;

A. Hashimoto, K. Hashimoto, hep-th/9909202, JHEP 9911(1999) 005;

K. Hashimoto, T. Hirayama, hep-th/0002090

[13] L. Jiang, "Dirac Monopole in Non-Commutative Space", hep-th/0001073

[14] R. Gopakumar, S. Minwala, J. Maldacena, A. Strominger, hep-th/0005048;

O. Ganor, G. Rajesh, S. Sethi, hep-th/00050046

[15] J. Harvey, P. Kraus, F. Larsen, E. Martinec, hep-th/0005031

[16] S. Gukov, I. Klebanov, A. Polyakov, hep-th/9711112, Phys. Lett. 423B (1998) 64-70

[17] N. Seiberg, L. Susskind, N. Toumbas, hep-th/0005040

[18] M. Kontsevich, "Deformation quantization of Poisson manifolds", q-alg/9709040

[19] A. Dhar, G. Mandal and S. R. Wadia, Mod. Phys. Lett. A7 (1992) 3129-3146;

A. Dhar, G. Mandal and S. R. Wadia, Int. J. Mod. Phys. A8 (1993) 3811-3828;

A. Dhar, G. Mandal and S. R. Wadia, Mod. Phys. Lett. A8 (1993) 3557-3568;

A. Dhar, G. Mandal and S. R. Wadia, Phys. Lett. 329B (1994) 15-26

[20] I. Bars, D. Minic, "Non-Commutative Geometry on a Discrete Periodic Lattice and Gauge Theory", hep-th/9910091

[21] W. Nahm, Phys. Lett. 90B (1980) 413;

W. Nahm, "The Construction of All Self-Dual Multimonopoles by the ADHM Method", in "Monopoles in quantum field theory", Craigie et al., Eds., World Scientific, Singapore (1982) ;

N.J. Hitchin, Comm. Math. Phys. 89 (1983) 145 
[22] D.-E. Diaconescu, Nucl. Phys. B503 (1997) 220-238, hep-th/9608163

[23] D. Bak, Phys. Lett. 471B (1999) 149-154, hep-th/9910135

[24] S. Moriyama, hep-th/0003231

[25] D. Mateos, "Noncommutative vs. commutative descriptions of D-brane BIons", hepth/0002020

[26] P. Etingof, I. Gelfand, V. Retakh, "Factorization of differential operators, quasideterminants, and nonabelian Toda field equations" q-alg/9701008

[27] R. Gopakumar, S. Minwala, A. Strominger, hep-th/0003160, JHEP 0005(2000) 020

[28] C. G. Callan, Jr., J. M. Maldacena, Nucl. Phys. B513 (1998) 198-212, hep-th/9708147 